\title{
The Moore-Penrose Generalized Inverse for Sums of Matrices
}

\author{
James Allen Fill* and \\ Donniell E. Fishkind ${ }^{\dagger}$ \\ September 18, 1998
}

Short title: Moore-Penrose Generalized Inverse for Sums

\begin{abstract}
In this paper we exhibit, under suitable conditions, a neat relationship between the Moore-Penrose generalized inverse of a sum of two matrices and the Moore-Penrose generalized inverses of the individual terms. We include an application to the parallel sum of matrices.
\end{abstract}

AMS 1991 subject classifications. Primary 15A09; secondary 15A18.

Key words and phrases. Moore-Penrose generalized inverse, Sherman-MorrisonWoodbury formula, singular value decomposition, rank additivity, parallel sum.

\footnotetext{
*Department of Mathematical Sciences, The Johns Hopkins University, Baltimore, Maryland 21218-2682, jimfill@jhu.edu. Research supported by NSF grants DMS-96-26756 and DMS-9803780.

$\dagger$ Department of Mathematics and Statistics, University of Southern Maine, Portland, Maine 04104, fishkind@payson.usm.maine.edu. Research carried out while this author was affiliated with The Johns Hopkins University, and supported by US Department of Education GAANN grant P200A40303.
} 


\section{Background and Main Result}

In the late 1940s and the 1950s Sherman and Morrison [11] [12], Woodbury [13], Bartlett [2], and Bodewig [4] discovered the following result. As in [7], $M_{m, n}$ denotes the space of complex-valued $m \times n$ matrices and, when $m=n$, this is shortened to $M_{n}$.

Theorem 1 (Sherman-Morrison-Woodbury) For $s \leq n$, let $A \in M_{n}$ and $G \in M_{s}$ both be invertible, and let $Y, Z \in M_{n, s}$. Then $A+Y G Z^{*}$ is invertible if and only if $G^{-1}+Z^{*} A^{-1} Y$ is invertible, in which case

$$
\left(A+Y G Z^{*}\right)^{-1}=A^{-1}-A^{-1} Y\left(G^{-1}+Z^{*} A^{-1} Y\right)^{-1} Z^{*} A^{-1} .
$$

The Sherman-Morrison-Woodbury (SMW) formula and related formulas are reviewed in Henderson and Searle [6]. The SMW formula has been used in a wide variety of applications; an excellent review by Hager [5] describes some of the applications to statistics, networks, structural analysis, asymptotic analysis, optimization, and partial differential equations.

In 1992, Riedel [10] proved an analogous formula (Theorem 2) for some cases where $A$ is singular. All matrices, including singular and even nonsquare matrices, have a Moore-Penrose generalized inverse. Given a matrix $A \in M_{m, n}$, the MoorePenrose generalized inverse of $A$, denoted $A^{\dagger}$, is the unique matrix in $M_{n, m}$ satisfying the conditions 


$$
\begin{aligned}
A A^{\dagger} A & =A, \\
A^{\dagger} A A^{\dagger} & =A^{\dagger},
\end{aligned}
$$

$A A^{\dagger}$ is Hermitian, and

$$
A^{\dagger} A \text { is Hermitian. }
$$

In particular, if $A=U \Sigma V^{*}$ is a singular value decomposition of $A$ (that is, if $U \in M_{m}$ and $V \in M_{n}$ are unitary and $\Sigma \in M_{m, n}$ has $\Sigma_{i, i} \geq 0$ for $1 \leq i \leq \min (m, n)$ and $\Sigma_{i, j}=0$ otherwise) then it may be verified (by checking (1)-(4)) that $A^{\dagger}=V \Sigma^{\dagger} U^{*}$, where $\Sigma^{\dagger}$ is defined by

$$
\Sigma_{i, j}^{\dagger}:=\left\{\begin{array}{cl}
\frac{1}{\Sigma_{i, i}} & \text { if } i=j \text { and } \Sigma_{i, i} \neq 0 \\
0 & \text { otherwise. }
\end{array}\right.
$$

Classical references on generalized inverses are [3] and [9].

Theorem 2 (Riedel) Let $s$ and $n$ be positive integers with $s \leq n ; A \in M_{n} ; G \in M_{s}$; $Y, Y_{p} \in M_{n, s} ; Z, Z_{p} \in M_{n, s}$. Assume $R(Y) \subseteq R(A), R\left(Y_{p}\right) \perp R(A), R(Z) \subseteq R\left(A^{*}\right)$, $R\left(Z_{p}\right) \perp R\left(A^{*}\right), G$ is invertible, $Y_{p}$ is of full rank, and $Z_{p}$ is of full rank. Assume also that $R\left(Y_{p}\right)=R\left(Z_{p}\right)$. Then

$$
\left(A+\left(Y+Y_{p}\right) G\left(Z+Z_{p}\right)^{*}\right)^{\dagger}=A^{\dagger}-D Z^{*} A^{\dagger}-A^{\dagger} Y C^{*}+D\left(G^{-1}+Z^{*} A^{\dagger} Y\right) C^{*}
$$

where $C:=Y_{p}\left(Y_{p}^{*} Y_{p}\right)^{-1}$ and $D:=Z_{p}\left(Z_{p}^{*} Z_{p}\right)^{-1}$.

The matrices $\left(Y+Y_{p}\right) G\left(Z+Z_{p}\right)^{*}$ in Theorem 2 and $Y G Z^{*}$ in Theorem 1 are referred to as the update matrices to the initial matrix A. A version of Riedel's theorem 
(Theorem 2) for the special case where we seek the Moore-Penrose generalized inverse of a rank-one update to the initial matrix can be found in [9].

Riedel verifies Theorem 2 by checking conditions (1)-(4). It must, however, be noted that the hypothesis $R\left(Y_{p}\right)=R\left(Z_{p}\right)$ of Theorem 2 is nowhere used in the verification of Theorem 2, and thus Theorem 2 is true without this part of the hypothesis. It is this key observation that allows us to make use of Theorem 2 in this paper. When we refer to Theorem 2 henceforth, we will be referring to this theorem without the aforementioned unnecessary hypothesis.

The purpose of this paper, given matrices $A$ and $B$ and suitable conditions, is to relate $(A+B)^{\dagger}$ cleanly to $A^{\dagger}$ and $B^{\dagger}$. This is done in Theorem 3, our main result, using Riedel's theorem. (For a subspace $\Omega$ we denote by $P_{\Omega}$ the orthogonal projection onto $\Omega$.)

Theorem 3 Let $A, B \in M_{n}$ with $\operatorname{rank}(A+B)=\operatorname{rank} A+\operatorname{rank} B$. Then

$$
\begin{aligned}
& \qquad \begin{aligned}
(A+B)^{\dagger}= & (I-S) A^{\dagger}(I-T)+S B^{\dagger} T \\
\text { where } \quad S & :=\left(P_{R\left(B^{*}\right)} P_{R\left(A^{*}\right)^{\perp}}\right)^{\dagger} \quad \text { and } \\
& T:=\left(P_{R(A)^{\perp}} P_{R(B)}\right)^{\dagger} .
\end{aligned}
\end{aligned}
$$

Example 4 Without the rank-additivity hypothesis $[\operatorname{rank}(A+B)=\operatorname{rank} A+\operatorname{rank} B]$, the conclusion of Theorem 3 is (in general) false. For example, let $A$ and $B$ be $1 \times 1$ matrices with 1 as their only entry. In the notation of Theorem 3, we compute 
$S=([1][0])^{\dagger}=[0]$ and $T=([0][1])^{\dagger}=[0]$. Hence,

$$
(I-S) A^{\dagger}(I-T)+S B^{\dagger} T=[1][1]^{\dagger}[1]+[0][1]^{\dagger}[0]=[1]
$$

while $(A+B)^{\dagger}=\left[\frac{1}{2}\right]$, contrary to the assertion of Theorem 3. But note rank $[2]=1 \neq$ $2=\operatorname{rank}[1]+\operatorname{rank}[1]$.

Is it possible, however, that the rank-additivity hypothesis in the statement of Theorem 3 can be eliminated in favor of a weaker condition? We show in Proposition 5 that the rank-additivity hypothesis cannot be avoided in any proof of Theorem 3 which employs Riedel's theorem (Theorem 2), since rank additivity is shown to be implied by the hypotheses of Riedel's theorem. (As mentioned, our proof of Theorem 3 relies on Theorem 2.)

For conditions when $\operatorname{rank}(A+B)=\operatorname{rank} A+\operatorname{rank} B$, see [8].

Remark. The matrices $S$ and $T$ appearing in (5) are far from determined by (5). For example, let $x$ and $y$ be orthonormal vectors in $\mathbf{C}^{n}$ with $n \geq 3$, and let

$$
A:=x x^{*}, \quad B:=y y^{*} .
$$

Applying Theorem 3 we obtain

$$
(A+B)^{\dagger}=\left(I-y y^{*}\right) A\left(I-y y^{*}\right)+\left(y y^{*}\right) B\left(y y^{*}\right)
$$

which simplifies to

$$
\left(x x^{*}+y y^{*}\right)^{\dagger}=x x^{*}+y y^{*} .
$$

But applying Theorem 3 with the roles of $A$ and $B$ reversed we obtain the different formula

$$
(A+B)^{\dagger}=\left(x x^{*}\right) A\left(x x^{*}\right)+\left(I-x x^{*}\right) B\left(I-x x^{*}\right)
$$


which, however, also simplifies to (6).

\subsection{Derivation of Main Result (Theorem 3)}

Our proof of Theorem 3 is based on the following proposition.

Proposition 5 Let $s$ and $n$ be positive integers with $s \leq n ; A \in M_{n} ; G \in M_{s}$; $Y, Y_{p} \in M_{n, s} ; Z, Z_{p} \in M_{n, s}$. Assume $R(Y) \subseteq R(A), R\left(Y_{p}\right) \perp R(A), R(Z) \subseteq R\left(A^{*}\right)$, and $R\left(Z_{p}\right) \perp R\left(A^{*}\right)$.

Of the following statements, 1 implies 2. Conversely, 2 and 3 imply 1.

1. $Y_{p}$ and $Z_{p}$ are of full rank.

2. $\operatorname{rank}\left[A+\left(Y+Y_{p}\right) G\left(Z+Z_{p}\right)^{*}\right]=\operatorname{rank} A+\operatorname{rank}\left[\left(Y+Y_{p}\right) G\left(Z+Z_{p}\right)^{*}\right]$.

3. $\operatorname{rank}\left[\left(Y+Y_{p}\right) G\left(Z+Z_{p}\right)^{*}\right]=s$.

Proposition 5 is used in proving Theorem 3, but it also demonstrates that rank additivity (of the initial matrix and the update matrix) is implied by the hypotheses of Theorem 2; since our proof of Theorem 3 relies on Theorem 2, the rank additivity hypothesis of Theorem 3 is, for us, unavoidable.

Proof of Proposition 5: Using the assumption $R(Y) \subseteq R(A)$ we find

$$
R\left[A+\left(Y+Y_{p}\right) G\left(Z+Z_{p}\right)^{*}\right] \subseteq R(A)+R(Y)+R\left(Y_{p}\right)=R(A)+R\left(Y_{p}\right) .
$$

Thus, if Statements 2 and 3 hold, then

$$
\operatorname{rank} A+s=\operatorname{rank}\left[A+\left(Y+Y_{p}\right) G\left(Z+Z_{p}\right)^{*}\right] \leq \operatorname{rank} A+\operatorname{rank} Y_{p}
$$


from which we conclude that $\operatorname{rank} Y_{p} \geq s$, that is, that $Y_{p}$ (and similarly $Z_{p}$ ) is of full rank (Statement 1).

Conversely, suppose $Y_{p}$ and $Z_{p}$ are of full rank (Statement 1). We have

$$
\operatorname{rank} Y_{p}=s \geq \operatorname{rank} G \geq \operatorname{rank}\left[\left(Y+Y_{p}\right) G\left(Z+Z_{p}\right)^{*}\right]
$$

In [10], Riedel points out that (when $Y_{p}$ and $Z_{p}$ are of full rank)

$$
\left[A+\left(Y+Y_{p}\right) G\left(Z+Z_{p}\right)^{*}\right]\left[A+\left(Y+Y_{p}\right) G\left(Z+Z_{p}\right)^{*}\right]^{\dagger}=A A^{\dagger}+Y_{p} Y_{p}^{\dagger}
$$

By the orthogonality of $R(A)$ and $R\left(Y_{p}\right)$, we have $\operatorname{rank}\left(A A^{\dagger}+Y_{p} Y_{p}^{\dagger}\right)=\operatorname{rank}\left(A A^{\dagger}\right)+$ $\operatorname{rank}\left(Y_{p} Y_{p}^{\dagger}\right)$. (Without loss of generality, $A A^{\dagger}$ and $Y_{p} Y_{p}^{\dagger}$ share the same unitary matrices in their singular value decompositions because of this orthogonality.) Thus,

$$
\begin{aligned}
\operatorname{rank}\left[A+\left(Y+Y_{p}\right) G\left(Z+Z_{p}\right)^{*}\right] & =\operatorname{rank}\left(A A^{\dagger}+Y_{p} Y_{p}^{\dagger}\right) \\
& =\operatorname{rank}\left(A A^{\dagger}\right)+\operatorname{rank}\left(Y_{p} Y_{p}^{\dagger}\right) \\
& =\operatorname{rank} A+\operatorname{rank} Y_{p} \\
& \geq \operatorname{rank} A+\operatorname{rank}\left[\left(Y+Y_{p}\right) G\left(Z+Z_{p}\right)^{*}\right]
\end{aligned}
$$

the last inequality holding by (7). Because (trivially)

$$
\operatorname{rank}\left[A+\left(Y+Y_{p}\right) G\left(Z+Z_{p}\right)^{*}\right] \leq \operatorname{rank} A+\operatorname{rank}\left[\left(Y+Y_{p}\right) G\left(Z+Z_{p}\right)^{*}\right]
$$

we conclude

$$
\operatorname{rank}\left[A+\left(Y+Y_{p}\right) G\left(Z+Z_{p}\right)^{*}\right]=\operatorname{rank} A+\operatorname{rank}\left[\left(Y+Y_{p}\right) G\left(Z+Z_{p}\right)^{*}\right]
$$

that is, Statement 2 holds. 
In proving Theorem 3 we will need also the following three facts about the MoorePenrose generalized inverse that can be verified directly from (1)-(4). For positive integers $t$ and $n$ such that $t \leq n$, let $L_{n, t}$ denote a matrix of size $n \times t$ with ones on the diagonal and zeros elsewhere. Let $r, s, p$, and $q$ be positive integers with $s \leq p$ and $r \leq q$, and let $A \in M_{r, s}, U \in M_{r}$, and $V \in M_{s}$ with $U$ and $V$ unitary. Then

$$
\left(L_{q, r} A L_{p, s}^{*}\right)^{\dagger}=L_{p, s} A^{\dagger} L_{q, r}^{*}
$$

and

$$
\left(U A V^{*}\right)^{\dagger}=V A^{\dagger} U^{*}
$$

If $A$ is of full rank with $r \geq s$, then

$$
A^{\dagger}=\left(A^{*} A\right)^{-1} A^{*}
$$

Proof of Theorem 3: To simplify notation, and since $n$ is fixed, we shorten $L_{n, t}$ to $L_{t}$ for $t \leq n$. Let $A$ and $B$ have respective singular value decompositions $U_{A} \Sigma_{A} V_{A}^{*}$ and $U_{B} \Sigma_{B} V_{B}^{*}$, where, without loss of generality, exactly the first $s$ diagonal entries of $\Sigma_{B}$ are nonzero and exactly the first $r$ diagonal entries of $\Sigma_{A}$ are zero.

Note that

$$
A+B=A+U_{B} L_{s} L_{s}^{*} \Sigma_{B} L_{s} L_{s}^{*} V_{B}^{*}=A+\left(Y+Y_{p}\right) G\left(Z+Z_{p}\right)^{*},
$$

where we define

$$
\begin{aligned}
G & :=L_{s}^{*} \Sigma_{B} L_{s}, \\
Y & :=P_{R(A)} U_{B} L_{s}=\left[U_{A}\left(I-L_{r} L_{r}^{*}\right) U_{A}^{*}\right] U_{B} L_{s},
\end{aligned}
$$




$$
\begin{aligned}
Y_{p} & :=P_{R(A)^{\perp}} U_{B} L_{s}=\left[U_{A} L_{r} L_{r}^{*} U_{A}^{*}\right] U_{B} L_{s}, \\
Z & :=P_{R\left(A^{*}\right)} V_{B} L_{s}=\left[V_{A}\left(I-L_{r} L_{r}^{*}\right) V_{A}^{*}\right] V_{B} L_{s}, \\
Z_{p} & :=P_{R\left(A^{*}\right)^{\perp}} V_{B} L_{s}=\left[V_{A} L_{r} L_{r}^{*} V_{A}^{*}\right] V_{B} L_{s} .
\end{aligned}
$$

Note that $G, Y, Y_{p}, Z$, and $Z_{p}$ satisfy all of the hypotheses of Theorem 2 since $Y_{p}$ and $Z_{p}$ are of full rank by Proposition 5 (because $\operatorname{rank} B=s$ and $\operatorname{rank}(A+B)=$ $\operatorname{rank} A+\operatorname{rank} B)$.

We next observe that (with $D$ and $C$ defined as in Theorem 2)

$$
\begin{aligned}
D G^{-1} C^{*} & =D L_{s}^{*} \Sigma_{B}^{\dagger} L_{s} C^{*} \\
& =D L_{s}^{*} V_{B}^{*} V_{B} \Sigma_{B}^{\dagger} U_{B}^{*} U_{B} L_{s} C^{*} \\
& =D L_{s}^{*} V_{B}^{*} B^{\dagger} U_{B} L_{s} C^{*} \\
& =D\left(Z^{*}+Z_{p}^{*}\right) B^{\dagger}\left(Y+Y_{p}\right) C^{*},
\end{aligned}
$$

and thus by Theorem 2 and (12) we have that

$$
(A+B)^{\dagger}=\left(I-D Z^{*}\right) A^{\dagger}\left(I-Y C^{*}\right)+\left(D Z^{*}+D Z_{p}^{*}\right) B^{\dagger}\left(Y C^{*}+Y_{p} C^{*}\right) .
$$

This is the basic form of $(A+B)^{\dagger}$ that we seek, and we proceed to compute $D Z^{*}$, $Y C^{*}, D Z_{p}^{*}$, and $Y_{p} C^{*}$

Because

$$
n \geq \operatorname{rank}(A+B)=\operatorname{rank} A+\operatorname{rank} B=n-r+s,
$$

we have $r \geq s$. By this, the fact that projection matrices are Hermitian and idempotent, and (8)-(10), we get

$$
Y C^{*}=Y\left(Y_{p}^{*} Y_{p}\right)^{-1} Y_{p}^{*}
$$




$$
\begin{aligned}
& =P_{R(A)} U_{B} L_{s}\left(L_{s}^{*} U_{B}^{*} P_{R(A)^{\perp}}^{*} P_{R(A)^{\perp}} U_{B} L_{s}\right)^{-1} L_{s}^{*} U_{B}^{*} P_{R(A)^{\perp}}^{*} \\
& =P_{R(A)} U_{B} L_{s}\left(L_{s}^{*} U_{B}^{*} P_{R(A)^{\perp}} U_{B} L_{s}\right)^{-1} L_{s}^{*} U_{B}^{*} P_{R(A)^{\perp}} \\
& =P_{R(A)} U_{B} L_{s}\left(L_{s}^{*} U_{B}^{*} U_{A} L_{r} L_{r}^{*} U_{A}^{*} U_{B} L_{s}\right)^{-1} L_{s}^{*} U_{B}^{*} U_{A} L_{r} L_{r}^{*} U_{A}^{*} \\
& =P_{R(A)} U_{B} L_{s}\left[\left(L_{r}^{*} U_{A}^{*} U_{B} L_{s}\right)^{*}\left(L_{r}^{*} U_{A}^{*} U_{B} L_{s}\right)\right]^{-1}\left(L_{r}^{*} U_{A}^{*} U_{B} L_{s}\right)^{*} L_{r}^{*} U_{A}^{*} \\
& =P_{R(A)} U_{B} L_{s}\left(L_{r}^{*} U_{A}^{*} U_{B} L_{s}\right)^{\dagger} L_{r}^{*} U_{A}^{*} \\
& =P_{R(A)} U_{B}\left(L_{r} L_{r}^{*} U_{A}^{*} U_{B} L_{s} L_{s}^{*}\right)^{\dagger} U_{A}^{*} \\
& =P_{R(A)}\left(U_{A} L_{r} L_{r}^{*} U_{A}^{*} U_{B} L_{s} L_{s}^{*} U_{B}^{*}\right)^{\dagger} \\
& =P_{R(A)}\left(P_{R(A)^{\perp}} P_{R(B)}\right)^{\dagger} \\
& =P_{R(A)} T
\end{aligned}
$$

and also

$$
\begin{aligned}
& D Z^{*}=Z_{p}\left(Z_{p}^{*} Z_{p}\right)^{-1} Z^{*} \\
& =P_{R\left(A^{*}\right)^{\perp}} V_{B} L_{s}\left(L_{s}^{*} V_{B}^{*} P_{R\left(A^{*}\right)^{\perp}}^{*} P_{R\left(A^{*}\right)^{\perp}} V_{B} L_{s}\right)^{-1} L_{s}^{*} V_{B}^{*} P_{R\left(A^{*}\right)}^{*} \\
& =P_{R\left(A^{*}\right)^{\perp}} V_{B} L_{s}\left(L_{s}^{*} V_{B}^{*} P_{R\left(A^{*}\right)^{\perp}} V_{B} L_{s}\right)^{-1} L_{s}^{*} V_{B}^{*} P_{R\left(A^{*}\right)} \\
& =V_{A} L_{r} L_{r}^{*} V_{A}^{*} V_{B} L_{s}\left[L_{s}^{*} V_{B}^{*} V_{A} L_{r} L_{r}^{*} V_{A}^{*} V_{B} L_{s}\right]^{-1} L_{s}^{*} V_{B}^{*} P_{R\left(A^{*}\right)} \\
& =V_{A} L_{r}\left(L_{r}^{*} V_{A}^{*} V_{B} L_{s}\right)\left[\left(L_{r}^{*} V_{A}^{*} V_{B} L_{s}\right)^{*}\left(L_{r}^{*} V_{A}^{*} V_{B} L_{s}\right)\right]^{-1} L_{s}^{*} V_{B}^{*} P_{R\left(A^{*}\right)} \\
& =V_{A} L_{r}\left(L_{r}^{*} V_{A}^{*} V_{B} L_{s}\right)^{* \dagger} L_{s}^{*} V_{B}^{*} P_{R\left(A^{*}\right)} \\
& =V_{A} L_{r}\left(L_{s}^{*} V_{B}^{*} V_{A} L_{r}\right)^{\dagger} L_{s}^{*} V_{B}^{*} P_{R\left(A^{*}\right)} \\
& =V_{A}\left(L_{s} L_{s}^{*} V_{B}^{*} V_{A} L_{r} L_{r}^{*}\right)^{\dagger} V_{B}^{*} P_{R\left(A^{*}\right)} \\
& =\left(V_{B} L_{s} L_{s}^{*} V_{B}^{*} V_{A} L_{r} L_{r}^{*} V_{A}^{*}\right)^{\dagger} P_{R\left(A^{*}\right)}
\end{aligned}
$$

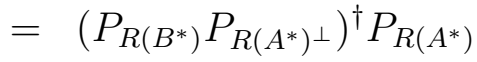




$$
=S P_{R\left(A^{*}\right)} .
$$

Similarly, we get

$$
\begin{aligned}
Y_{p} C^{*} & =P_{R(A)^{\perp}} T \quad \text { and } \\
D Z_{p}^{*} & =S P_{R\left(A^{*}\right)^{\perp} .}
\end{aligned}
$$

By plugging (14)-(16) into (13), and noting that $P_{R\left(A^{*}\right)} A^{\dagger}=A^{\dagger}$ and $A^{\dagger} P_{R(A)}=A^{\dagger}$, the assertion of Theorem 3 follows.

\section{Application to the Parallel Sum}

It is well known in elementary electronics that if two resistors with resistances $r_{1}$ and $r_{2}$ are placed in parallel, then the cumulative resistance $r$ is computed by the formula

$$
r=r_{1}\left(r_{1}+r_{2}\right)^{-1} r_{2}=\left(\frac{1}{r_{1}}+\frac{1}{r_{2}}\right)^{-1} .
$$

With the idea of generalizing this notion to matrices, Anderson and Duffin [1] define, for $A, B \in M_{n}$, the parallel sum of $A$ and $B$ as

$$
A: B:=A(A+B)^{\dagger} B,
$$

which, in the case that $A$ and $B$ are (scalar) resistances, is exactly the formula in (17). An alternative definition for the parallel sum of $A$ and $B$ can be found in Rao and Mitra [9], where it is defined as

$$
A \| B:=\left(A^{\dagger}+B^{\dagger}\right)^{\dagger},
$$


which, in the case that $A$ and $B$ are (scalar) resistances, is again exactly the formula in (17). Given some assumptions on $A$ and $B$, [9] presents necessary and sufficient conditions for the two definitions of parallel sum to agree.

The following result uses Theorem 3 to provide, under certain conditions, a neat equation relating $A \| B$ to $A$ and $B$.

Corollary 6 Let $A, B \in M_{n}$ with $\operatorname{rank}(A \| B)=\operatorname{rank} A+\operatorname{rank} B$. Then

$$
\begin{aligned}
A \| B & =(I-R) A(I-W)+R B W \\
\text { where } R & :=\left(P_{R(B)} P_{R(A)^{\perp}}\right)^{\dagger} \text { and } \\
W & :=\left(P_{R\left(A^{*}\right)^{\perp}} P_{R\left(B^{*}\right)}\right)^{\dagger} .
\end{aligned}
$$

Corollary 6 is an immediate corollary of Theorem 3, where $A^{\dagger}$ and $B^{\dagger}$ of Theorem 6 play the roles of $A$ and $B$ in Theorem 3 .

Acknowledgements. The authors thank Adi Ben-Israel, Shih-Ping Han, Roger Horn, and Kurt Riedel for valuable discussions and two anonymous referees for their helpful suggestions.

\section{References}

[1] W. N. Anderson, Jr. and R. J. Duffin, Series and parallel addition of matrices, Journal of Mathematical Analysis and Applications 26 (1969), 576-594.

[2] M. S. Bartlett, An inverse matrix adjustment arising in discriminant analysis, Ann. Math. Statist. 22 (1951), 107-111. 
[3] A. Ben-Israel and T. Greville, Generalized Inverses, Theory and Applications, Wiley, New York, 1974.

[4] E. Bodewig, Matrix Calculus, North Holland, Amsterdam, 1959.

[5] W. W. Hager, Updating the inverse of a matrix, SIAM Review 31 (1989), 221239.

[6] H. V. Henderson and S. R. Searle, On deriving the inverse of a sum of matrices, SIAM Review 23 (1981), 53-60.

[7] R. A. Horn and C. R. Johnson, Matrix Analysis, Cambridge University Press, 1985.

[8] G. Marsaglia and G. P. H. Styan, When $\operatorname{does} \operatorname{rank}(A+B)=\operatorname{rank} A+\operatorname{rank} B$ ?, Canadian Mathematical Bulletin 15 (1972), 451-452.

[9] C. R. Rao and S. K. Mitra, Generalized Inverse of Matrices and its Applications, Wiley, New York, 1971.

[10] K. S. Riedel, A Sherman-Morrison-Woodbury identity for rank augmenting matrices with application to centering, SIAM J. Matrix Anal. Appl. 13 (1992), 659-662.

[11] J. Sherman and W. J. Morrison, Adjustment of an inverse matrix corresponding to changes in the elements of a given column or given row of the original matrix, Ann. Math. Statist. 20 (1949), 621. 
[12] J. Sherman and W. J. Morrison, Adjustment of an inverse matrix corresponding to a change in one element of a given matrix, Ann. Math. Statist. 21 (1950), 124-127.

[13] M. A. Woodbury, Inverting modified matrices, Technical Report 42, Statistical Research Group, Princeton University, Princeton, NJ (1950). 